\title{
Prostate-specific membrane antigen positron emission tomography (PSMA-PET) for local staging of prostate cancer: a systematic review and meta-analysis
}

\author{
Sungmin Woo ${ }^{1 *} \mathbb{D}$, Soleen Ghafoor ${ }^{1}$, Anton S. Becker ${ }^{1}$, Sangwon Han², Andreas G. Wibmer', Hedvig Hricak',
} Irene A. Burger ${ }^{3,4}$, Heiko Schöder ${ }^{1}$ and Hebert Alberto Vargas ${ }^{1}$

\author{
* Correspondence: woos@mskcc.org \\ 'Department of Radiology, \\ Memorial Sloan Kettering Cancer \\ Center, 1275 York Avenue, New \\ York, NY 10065, USA \\ Full list of author information is \\ available at the end of the article
}

\begin{abstract}
Purpose: Prostate-specific membrane antigen positron emission tomography (PSMAPET) has shown promise for detecting nodal and distant prostate cancer (PCa) metastases. However, its performance for local tumor staging is not as well established. The purpose of this study was to review the diagnostic performance of PSMA-PET for determining seminal vesical invasion (SVI) and extraprostatic extension (EPE).

Methods: Pubmed and Embase databases were searched until January 12, 2020. Studies assessing accuracy of PSMA-PET in determining SVI and EPE were included. Study quality was evaluated with the revised Quality Assessment of Diagnostic Accuracy Studies-2 tool. Pooled sensitivity and specificity were calculated using hierarchical summary receiver operating characteristics modeling. Heterogeneity was explored using meta-regression analyses for anatomical imaging component (MRI vs CT) and by testing for a threshold effect.

Results: Twelve studies (615 patients) were included. Pooled sensitivity and specificity were 0.68 (95\% Cl 0.53-0.81) and 0.94 (95\% Cl 0.90-0.96) for SVI and 0.72 (95\% Cl 0.56-0.84) and 0.87 (95\% Cl 0.72-0.94) for EPE. Meta-regression analyses showed that for SVI, PET/MRI demonstrated greater sensitivity than PET/CT (0.87 [95\% Cl 0.75-0.98] vs 0.60 [95\% Cl 0.47-0.74]; $p=0.02$ for joint model) while specificity was comparable (0.91 [95\% Cl 0.84-0.97] vs. 0.96 [95\% Cl 0.93-0.99]) but not for EPE ( $p=$ 0.08). A threshold effect was present for studies assessing EPE (correlation coefficient $=0.563[95 \% \mathrm{Cl},-0.234-0.908]$ between sensitivity and false-positive rate).
\end{abstract}

Conclusion: PSMA-PET has moderate sensitivity and excellent specificity for assessing local tumor extent in patients with PCa. PET/MRI showed potential for greater sensitivity than PET/CT in assessing SVI.

Keywords: Prostate-specific membrane antigen, Positron emission tomography, Prostate cancer, Computed tomography, Magnetic resonance imaging, Meta-analysis 


\section{Introduction}

Prostate cancer is the second most common cancer and the 5th leading cause of cancer-related deaths worldwide (Bray et al. 2018). Local staging and identification of nodal and distant metastases are important in determining the most appropriate management strategy. In surgical candidates planning to undergo radical prostatectomy, interrogating for the presence of seminal vesical invasion (SVI) and extraprostatic extension (EPE) is key, as they are associated with adverse oncological outcomes such as biochemical recurrence, metastasis, and worse survival (Mikel Hubanks et al. 2014). In addition, patients without EPE can undergo nerve-sparing surgery with the aim of reducing postoperative functional morbidity including urinary incontinence and erectile dysfunction (Mottet et al. 2017).

Nomograms combining clinicopathological information including prostate-specific antigen (PSA) levels, clinical stage based on digital rectal examination, and biopsyrelated information (Gleason score, number, and percentage of positive cores) are often used to predict the extent of prostate cancer (Ohori et al. 2004). However, there is an increasing number of studies showing that incorporating preoperative magnetic resonance imaging (MRI) results provide incremental value in predicting SVI and EPE (Ohori et al. 2004; Nyarangi-Dix et al. 2018; Mehralivand et al. 2019; Park et al. 2020). Nevertheless, these results are still imperfect with area under the curves (AUC) ranging from 0.74-0.87 (Jansen et al. 2019; Wang et al. 2007; Weaver et al. 2018). A metaanalysis including over 9700 patients confirmed that the sensitivity of MRI for SVI and EPE is limited and heterogeneous among different studies with 57\% (confidence interval (CI) $0.49-0.64$ ) and 58\% (CI 0.47-0.68), respectively. Therefore, there is an unmet clinical need to improve preoperative risk assessment in patients with prostate cancer.

Prostate-specific membrane antigen (PSMA) positron emission tomography (PET) is a relatively novel imaging technique, which targets PSMA, a transmembrane protein expressed on prostate cells with levels of expression increasing with greater degree of dysplasia (Bostwick et al. 1998; Hofman et al. 2018). Over the past few years, evidence has accumulated regarding the utility of PSMA-PET, especially those using ${ }^{68}$ Gallium $\left({ }^{68} \mathrm{Ga}\right)$-based radioligands. It is now recognized that this novel imaging modality is excellent in determining sites of disease in the biochemically recurrent post-treatment setting, identifying lymph node and bone metastases, and even in detecting the dominant lesion for primary staging, with these translating to actual impact in the management of patients (Fendler et al. 2019; Eiber et al. 2016; Perera et al. 2020; Zhou et al. 2019; Corfield et al. 2018; Han et al. 2018). However, the diagnostic performance of PSMA-PET in determining local disease extent is not well established as there are only scattered small-scaled reports in the literature. Therefore, the purpose of this study was to systematically review the literature and meta-analyze the diagnostic performance of PSMA PET for determining SVI and EPE based on radical prostatectomy as the reference standard.

\section{Materials and methods}

\section{Search strategy and study selection}

This study was performed according to the Preferred Reporting Items for Systematic Reviews and Meta-Analyses (PRISMA) guidelines (Liberati et al. 2009). Pubmed and 
Embase databases were systematically searched from inception until January 12, 2020, using keywords and related terms of "prostate", "PSMA-PET", "SVI", and "EPE" based on the search query as the following: (prostate OR prostatic) AND ("prostate-specific membrane antigen" OR PSMA) AND ("positron emission" OR PET) AND ("extracapsular extension" OR ECE OR "extraprostatic extension" OR EPE OR "seminal vesical invasion" OR SVI OR T3 OR T3a OR T3b OR ((local OR localized OR regional OR locoregional) AND (stage OR staging OR extent* OR invasion))). The reference lists of eligible articles were also scrutinized to further identify relevant articles. No language limitations were applied.

Studies were included based on "Patient, Index test, Comparator, Outcome, and Study design" (PICOS) criteria: (1) "patients" with prostate cancer presenting for primary staging; (2) PSMA-PET as "index test;" (3) radical prostatectomy as the "comparator" or reference standard; (4) SVI or EPE as the "outcome;" and (5) "study design" of clinical trials, prospective or retrospective cohort studies either published as original articles or conference abstracts. Of note, we planned to only meta-analyze studies assessing ${ }^{68} \mathrm{Ga}$-based radioligands as they are widely used and investigated in the literature.

Studies were excluded if they (1) included a small number of patients $(<10),(2)$ were of other publication types (e.g., review articles, letters, or editorials); (3) focused on other topics; (4) did not provide sufficient data to calculate $2 \times 2$ contingency tables with regard to sensitivity and specificity; or (5) had overlap in the study population. When overlap was present, we used the study with more comprehensive information required for meta-analysis.

The study selection process was performed by two independent reviewers (S.W. and S.G.) and discussion with a third reviewer (H.A.V.) was performed when there was disagreement.

\section{Data extraction and quality assessment}

Relevant study-, clinicopathological-, and PET-related information were extracted and collated in Excel 2016 as follows: (1) study: first author, publication year, institution, period of enrollment, country of origin, study design (prospective vs. retrospective), and endpoint (SVI, EPE, or both); (2) clinicopathological: number of patients, age, serum PSA level, Gleason score, risk classification (Mottet et al. 2020), (3) PET: vendor, type of scanner, ligands, anatomical imaging component (MRI vs. CT), and whether PET was assessed blinded to clinicopathological information or not.

The quality of the studies was assessed using the revised Quality Assessment of Diagnostic Accuracy Studies-2 (QUADAS-2) tool (Whiting et al. 2011). Data extraction and quality assessment were performed by the same three reviewers above in the same manner.

\section{Data synthesis and analysis}

The primary outcome of our study was to assess the diagnostic performance of PSMAPET for determining SVI and EPE in terms of sensitivity and specificity. The secondary outcome was to evaluate whether there are differences in the performance between $\mathrm{PET} / \mathrm{MRI}$ and PET/CT. 
True positive, false negative, false positive, and true negative values were tabulated using sensitivity and specificity or the corresponding raw data provided from each of the included studies. If multiple diagnostic test accuracy results by multiple readers were given within a study, the average value across all readers was used. Sensitivity and specificity were meta-analytically pooled using hierarchical logistic regression modeling and corresponding hierarchical summary ROC (HSROC) curves were generated with their 95\% confidence and prediction regions (Suh and Park 2016; Lee et al. 2015). Publication bias was evaluated by subjective assessment of the Deeks' funnel plot and based on the $p$ value of Deeks' asymmetry test (Deeks et al. 2005).

Heterogeneity was assessed with several methods. First, heterogeneity was evaluated using the Cochran's $Q$ test. Second, Higgins $I^{2}$ test was used to determine the degree of heterogeneity as follows: inconsistency index $\left(I^{2}\right)=0-40 \%$, unimportant; 30-60\%, moderate; $50-90 \%$, substantial; and 75-100\%, considerable (Higgins and Green 2011). Third, we tested for the presence of a threshold effect, which means a positive correlation between the sensitivity and false-positive rate. Finally, meta-regression analysis was performed using anatomical imaging component of the PET (MRI vs. CT) as a covariate to ascertain if there were differences in the diagnostic performance between studies using PET/MRI and PET/CT.

The "metandi" and "midas" modules in Stata 10.0 (StataCorp LP, College Station, TX, USA) and "mada" package in the $R$ software version 3.6.1 (R Foundation for Statistical Computing, Vienna, Austria) were used for statistical analyses. A two-tailed $P<0.05$ was considered statistically significant with the exception of Deeks' asymmetry test, where $<0.1$ indicated statistical significance.

\section{Results}

\section{Literature search}

Initially, 592 articles were identified from the systematic search. After removal of 106 duplicates, 460 articles were further excluded by screening the titles and abstracts. Fulltext reviews were done on the remaining 26 articles, among which 16 studies were excluded owing to the following reasons: non- ${ }^{68} \mathrm{Ga}$-based radioligands $\left({ }^{18} \mathrm{~F}-\mathrm{PSMA}-1007\right)$ was used $(n=1)$, PSMA PET was correlated with clinical staging $(n=4)$, inter-observer agreement study $(n=2)$, an agreement between PET/MRI and PET/CT $(n=1)$, assessment of utility of CT urography together with PET $(n=1)$, comparison of standardized uptake value between tumor and nontumor $(n=1)$, no evaluation of local staging $(n=$ 1 ), insufficient data for reconstructing $2 \times 2$ tables $(n=2)$, and overlap in patient population $(n=3)$. Two additional articles were found upon additional screening of the reference lists. Finally, 12 studies including a total of 615 patients were included (Agrawal et al. 2017; Berger et al. 2018; Dekalo et al. 2019; Fendler et al. 2016; Gao et al. 2019; Grubmuller et al. 2018; Gupta et al. 2018; Muehlematter et al. 2019; Thalgott et al. 2018; van Leeuwen et al. 2019; von Klot et al. 2017; Yilmaz et al. 2019). All 12 assessed SVI while 8 of them evaluated EPE. The study selection process is shown in Fig. 1.

\section{Characteristics of included studies}

The characteristics of the included studies are summarized in Tables 1 and 2. In brief, all studies were retrospective single-center studies except for one prospective single 


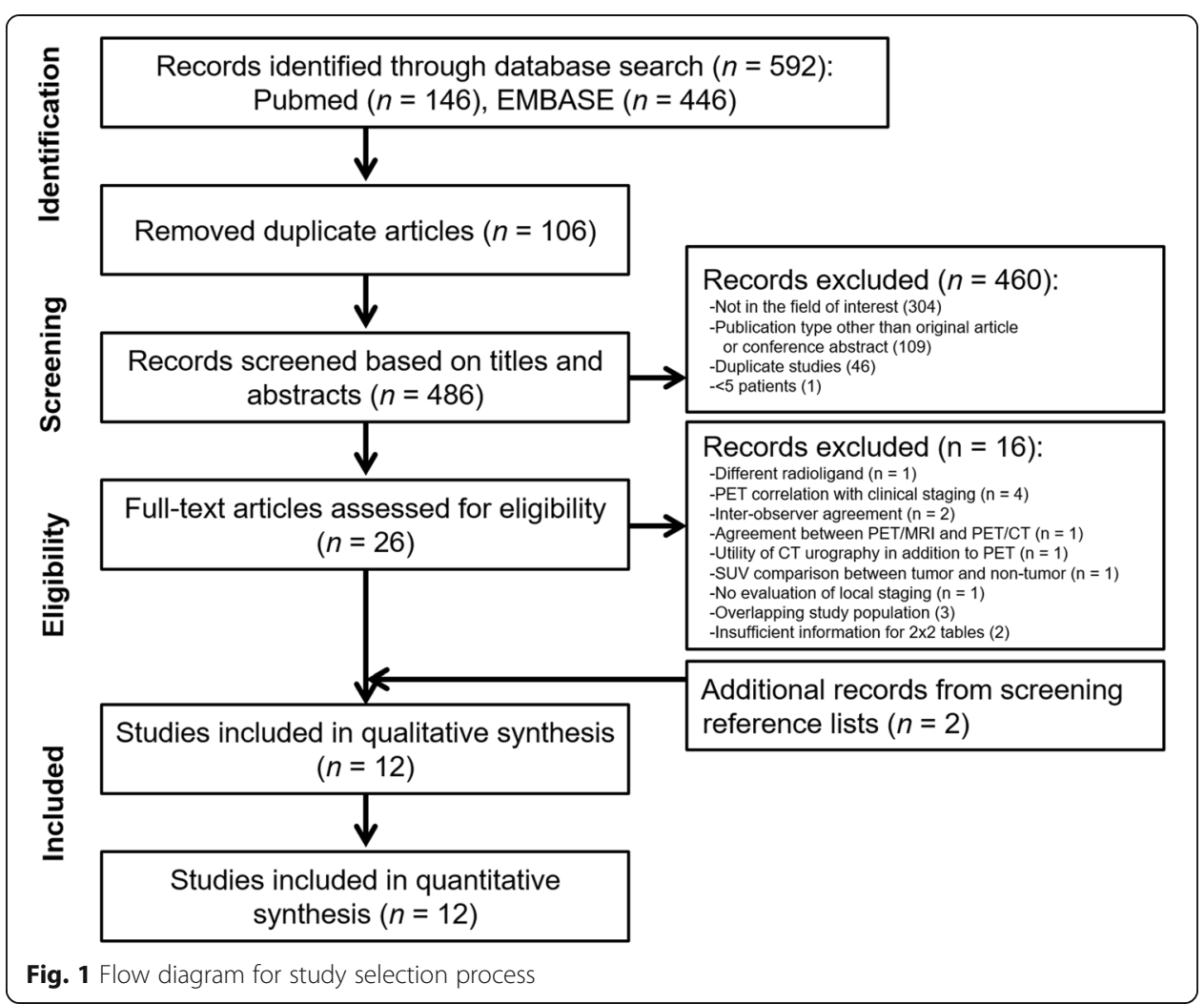

center (Grubmuller et al. 2018) and one retrospective dual center study (van Leeuwen et al. 2019). The number of patients ranged from 21 to 140 with median ages ranging from 63 to 70 years. Median PSA levels were 7.6-58.7 ng/mL and the median Gleason scores were 7-9. Two studies only included patients with high risk, 7 with intermediate to high risk, and 2 with low to high risk (which were predominantly constituted with intermediate to high-risk patients). Eleven studies used ${ }^{68} \mathrm{Ga}$-PSMA-11 and one used ${ }^{68} \mathrm{Ga}$-PSMA-I\&T. Anatomical imaging was based on MRI in 3 studies and CT in 9.

\section{Quality assessment}

All studies were of moderate to good quality, satisfying at 4 or more of the 7 domains in the QUADAS-2 tool except for one which only met 3 domains (Fig. 2). In the patient selection domain, 2 studies had unknown risk of bias as it was not clear whether the patient enrollment was consecutive or not (Agrawal et al. 2017; von Klot et al. 2017). One study had high concern for applicability as minority of the patients $(2 / 50)$ had rising PSA after radiation treatment and these patients could not be separately analyzed from the other 48 with newly diagnosed prostate cancer (Berger et al. 2018). Regarding the index test domain, six studies had unknown risk of bias and concern for applicability as there were no clear criteria for interpreting SVI and EPE (Agrawal et al. 2017; Berger et al. 2018; Dekalo et al. 2019; Fendler et al. 2016; Gao et al. 2019; van Leeuwen et al. 2019; Yilmaz et al. 2019). One additional study which did not have clear criteria and therefore unclear concern for applicability, had high risk of bias as the interpretation of PSMA-PET was performed without blinding to the surgico-pathological 


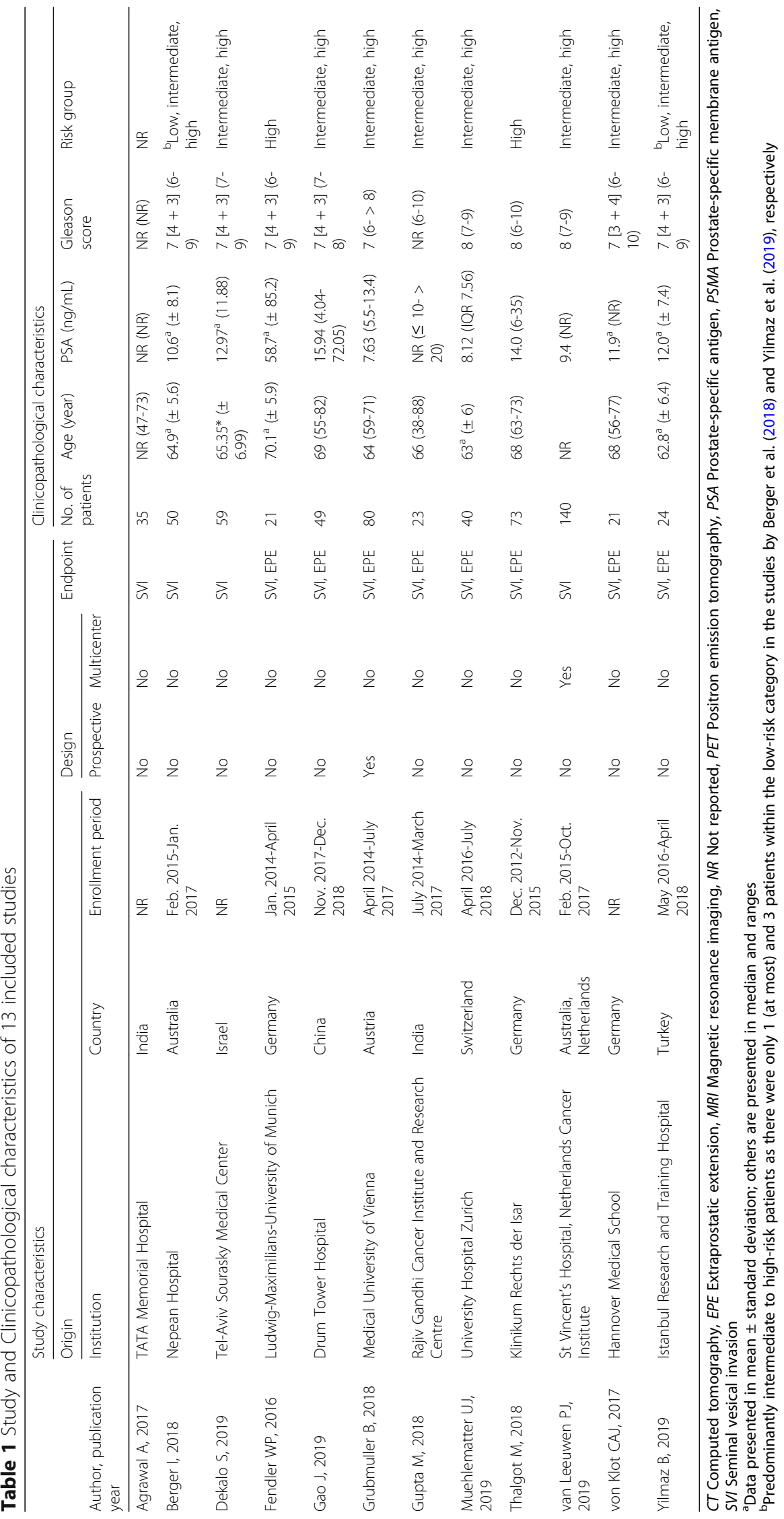




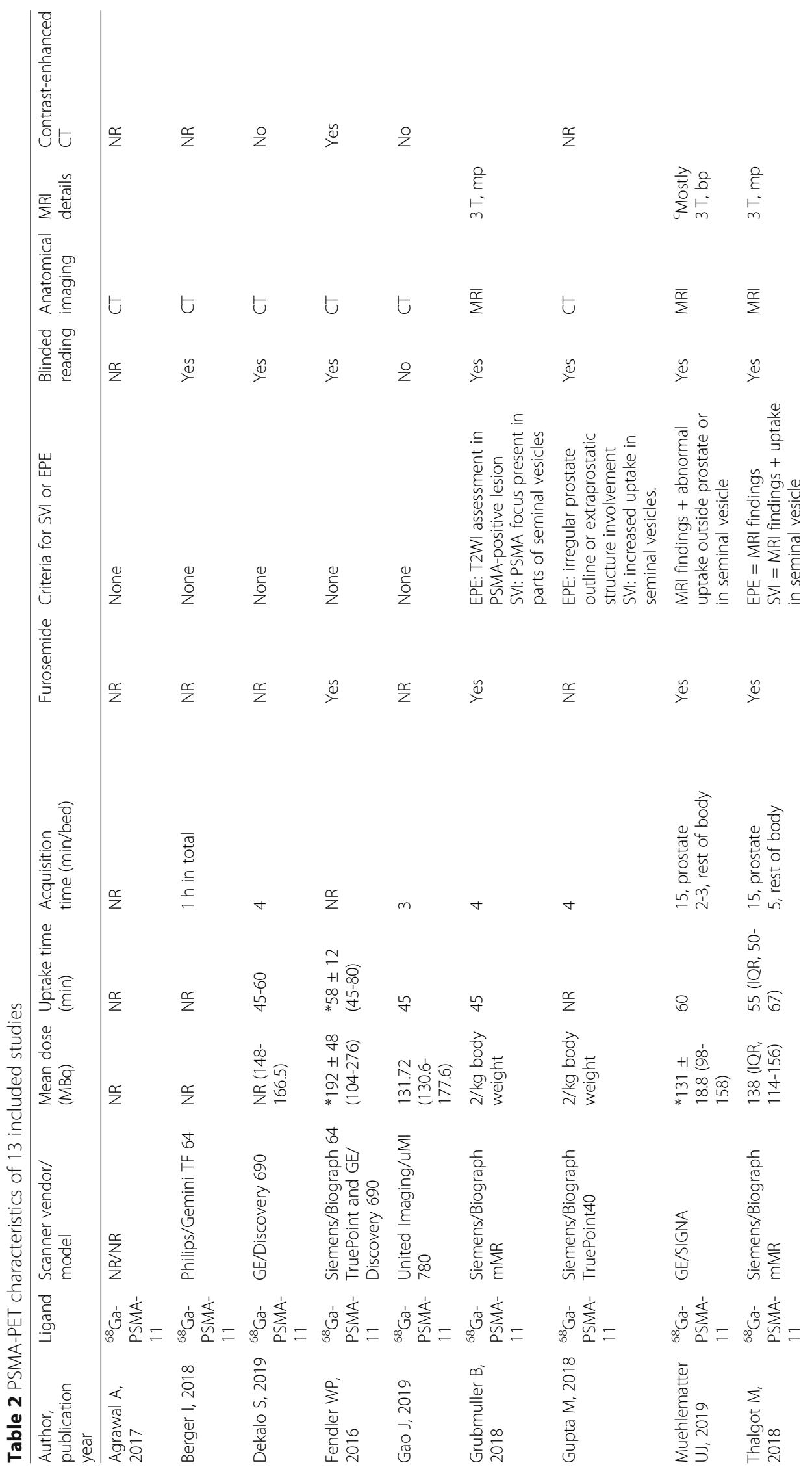




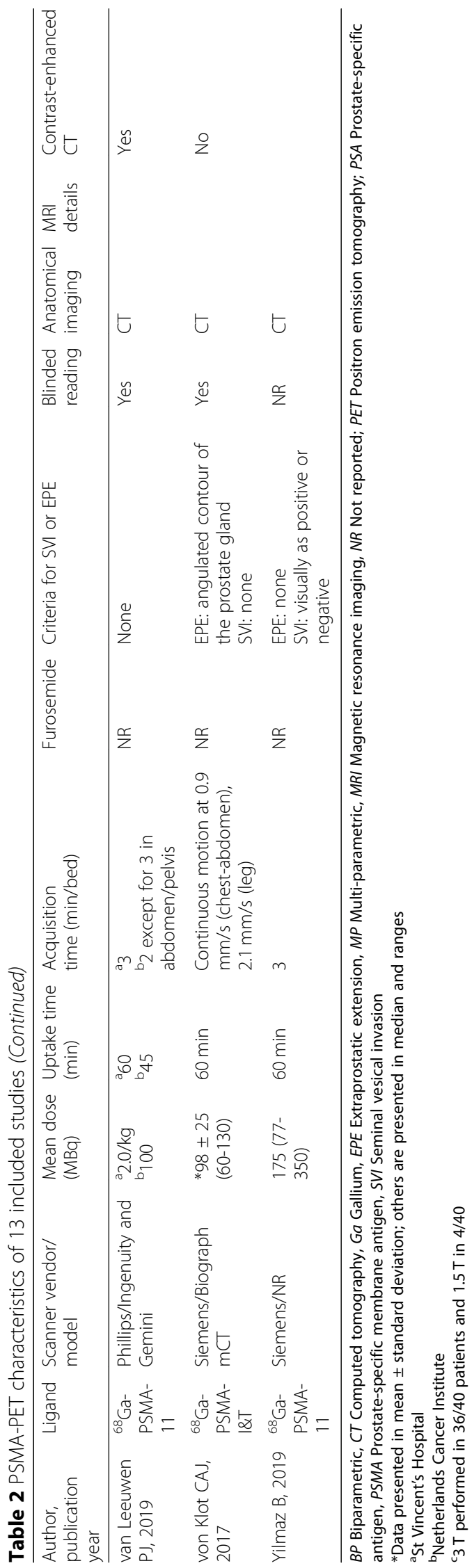




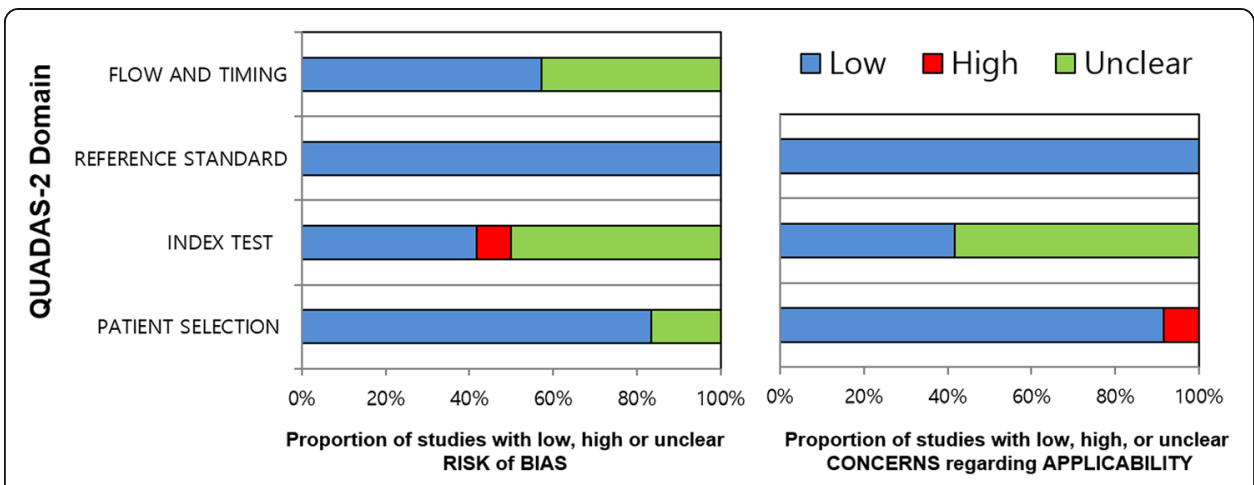

Fig. 2 Grouped bar charts for QUADAS-2 tool summarizing risk of bias and concern for applicability of the 12 included studies

reference standard [30]. In the reference standard domain, all studies were at low risk of bias and concern for applicability. In the flow and timing domain, 6 studies had unknown risk of bias as the interval between PSMA-PET and prostatectomy was not provided (Agrawal et al. 2017; Dekalo et al. 2019; Grubmuller et al. 2018; Gupta et al. 2018; van Leeuwen et al. 2019; von Klot et al. 2017).

\section{Diagnostic performance of PSMA PET for SVI}

The summary sensitivity and specificity were 0.69 (95\% CI $0.53-0.81)$ and 0.94 (95\% CI 0.90-0.96), respectively (Fig. 3). The area under the HSROC curve was 0.94 (95 \% CI 0.92-0.96). No publication bias was suggested in the Deeks' funnel plot ( $p=0.46$ for slope coefficient) (Fig. 4). The $Q$ test indicated that heterogeneity was present $(p=$ $0.007)$, which was substantial and moderate for sensitivity $\left(I^{2}=68 \%\right)$ and specificity $\left(I^{2}\right.$ $=47 \%)$, respectively, based on the Higgin's $I^{2}$ test. The coupled forest plot did not show a threshold effect (Fig. 5) with no demonstrable correlation between sensitivity and false-positive rate (correlation coefficient $=0.014$ [95\% CI, -0.564-0.583]). At meta-

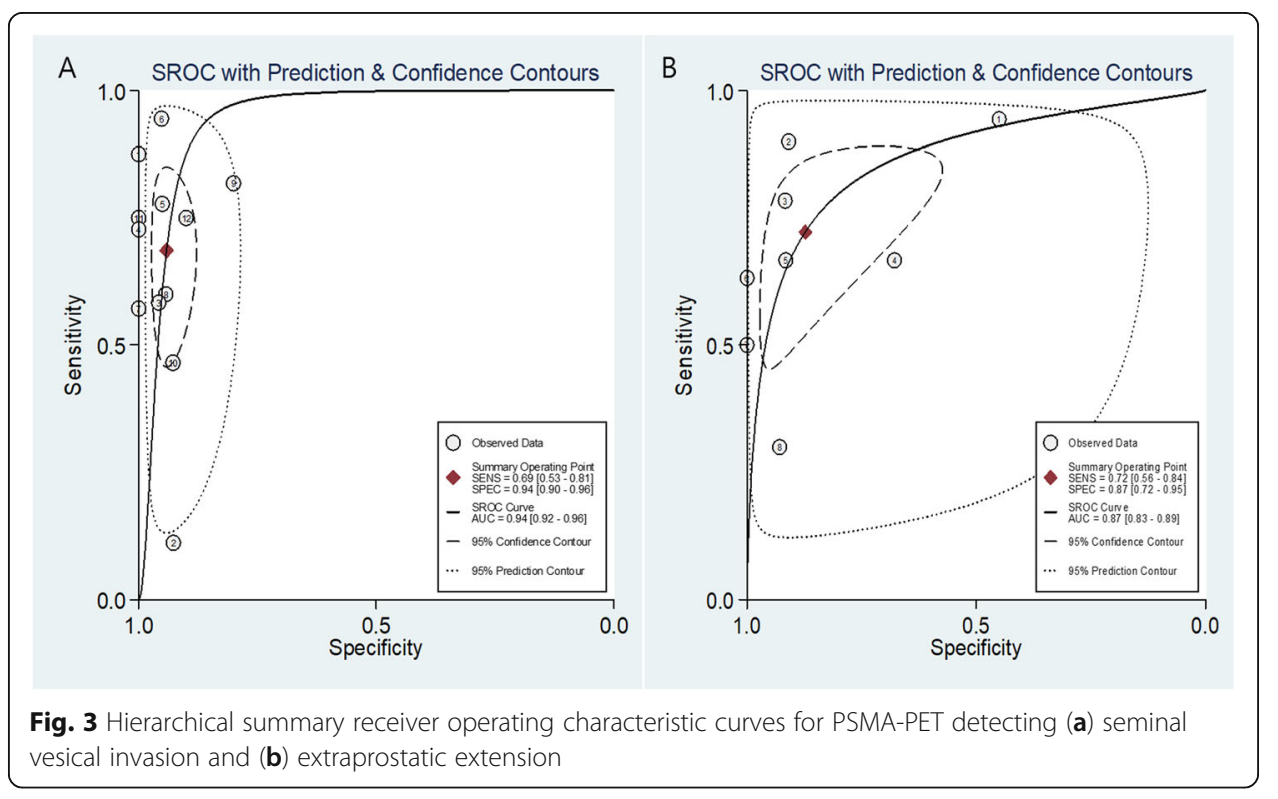



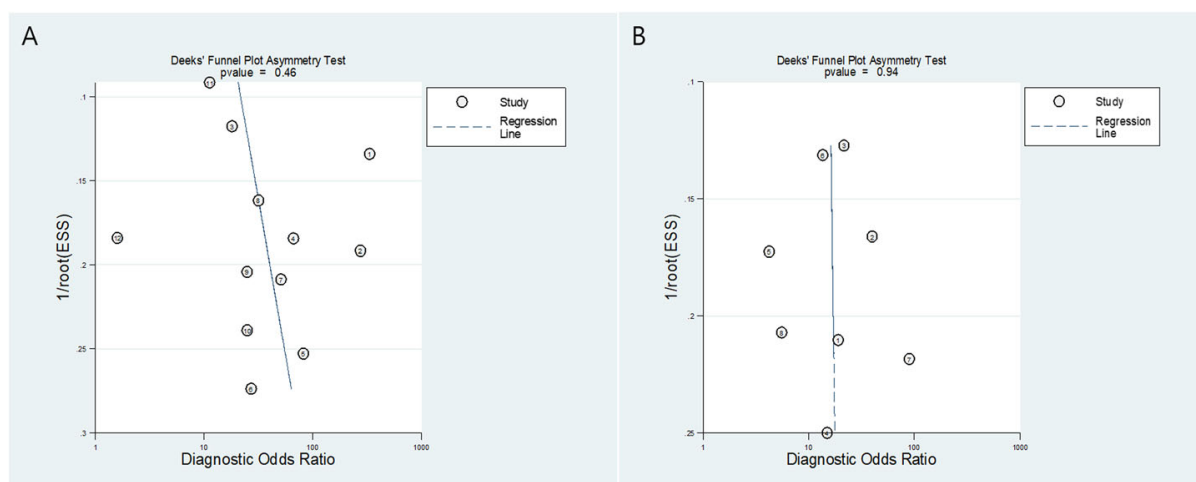

Fig. 4 Deeks' funnel plot. $P$ values of 0.46 and 0.94 for studies assessing (a) seminal vesical invasion and (b) extraprostatic extension indicate absence of publication bias

regression analysis, anatomical imaging modality was a source of heterogeneity $(p=$ 0.02) with PET/MRI showing significantly greater sensitivity (0.87 [95\% CI 0.75-0.98]) for detecting SVI compared with PET/CT (0.60 [95\% CI 0.47-0.74]) while the specificity was comparable (0.91 [95\% CI 0.84-0.97] vs. 0.96 [95\% CI 0.93-0.99], respectively).

\section{Diagnostic performance of PSMA PET for EPE}

The summary sensitivity and specificity were 0.72 (95\% CI $0.56-0.84)$ and 0.87 (95\% CI 0.72-0.94), respectively (Fig. 3). The area under the HSROC curve was 0.87 (95\% CI $0.83-0.89$ ). No publication bias was suggested in the Deeks' funnel plot $(p=0.94$ for slope coefficient) (Fig. 4). The $Q$ test indicated that heterogeneity was present $(p<$ $0.001)$, which was substantial for both sensitivity $\left(I^{2}=76 \%\right)$ and specificity $\left(I^{2}=78 \%\right)$, respectively. A threshold effect was suggested based on the coupled forest plots (Fig. 5) with a positive correlation between sensitivity and false-positive rate (correlation coefficient $=0.563$ [95\% CI, -0.234-0.908]). At meta-regression analysis, anatomical imaging modality was not a significant factor of heterogeneity $(\mathrm{p}=0.08)$. Studies using PET/ MRI demonstrated summary sensitivity and specificity of 0.82 [95\% CI 0.67-0.97] and

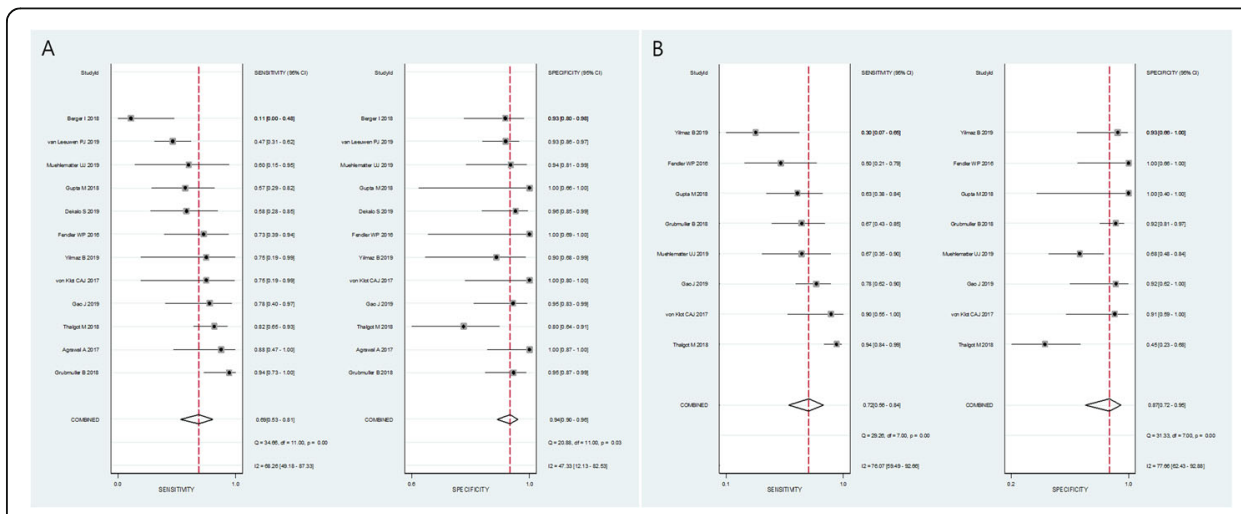

Fig. 5 Coupled forest plots of sensitivity and specificity for (a) seminal vesical invasion and (b) extraprostatic extension. Numbers are pooled estimates with $95 \%$ confidence intervals (Cl) in parentheses and heterogeneity statistics are shown at the bottom right. Horizontal lines indicate $95 \% \mathrm{Cls}$ 
0.73 [95\% CI 0.52-0.94], respectively; whereas for studies using PET/CT, they were 0.65 [95\% CI 0.47-0.83] and 0.95 [95\% CI 0.89-1.00], respectively.

\section{Discussion}

In the current meta-analysis, we evaluated the diagnostic performance of PSMA-PET in detecting SVI and EPE in patients with newly diagnosed prostate cancer treated with radical prostatectomy. We found that PSMA-PET had moderate sensitivity and excellent specificity for both SVI and EPE. It has already been well recognized in the literature that PSMA-PET shows good performance in detecting and localizing the primary tumor along with its excellent ability to detect metastases in the regional nodes, bones, and soft tissues. The addition of accurate assessment of the local extent of prostate cancer reported herein provides additional rationale for PSMA-PET to be used as a "one-stop-shop" imaging modality in the primary staging of prostate cancer.

There was moderate to substantial heterogeneity among the studies using PSMAPET to assess SVI. We were able to ascertain that one major source of heterogeneity was whether MRI or CT was used as the anatomical imaging component of PSMAPET. The three studies (Grubmuller et al. 2018; Muehlematter et al. 2019; Thalgott et al. 2018) using PET/MRI showed significantly greater sensitivity (0.87 vs. 0.60 ) with similar specificity (0.91 vs. 0.96) compared with the other nine studies (Agrawal et al. 2017; Berger et al. 2018; Dekalo et al. 2019; Fendler et al. 2016; Gao et al. 2019; Gupta et al. 2018; van Leeuwen et al. 2019; Yilmaz et al. 2019) using PET/CT. The superior sensitivity of PSMA-PET/MRI compared with PSMA-PET/CT can be attributed to the synergistic effect of combining the MRI findings with the functional information from PSMA-PET. All three studies using PET/MRI were performed at 3-Tesla scanners with biparametric protocol in one (Muehlematter et al. 2019) and multiparametric protocol in the remaining two studies, which was shown to be helpful for increasing the sensitivity of detecting SVI in a prior meta-analysis by de Rooij et al. (de Rooij et al. 2016). In addition, PET/MRI potentially enhances the detection of the primary tumor itself compared with multiparametric MRI (Eiber et al. 2016; Hicks et al. 2018; Park et al. 2018). On the contrary, CT on its own has a limited role in detecting the tumor and assessing the local extent, rather simply provides an anatomical correlate for assessing the areas of tracer uptake on the PSMA-PET. Nevertheless, it should be noted that there were only three studies using PET/MRI and they were indirectly compared with other studies using PET/CT. Further larger studies performing a head-to-head comparison between PET/MRI and PET/CT are needed to validate the potential superiority of using MRI over CT in determining SVI.

Substantial heterogeneity was also noted among studies assessing EPE, and at least part of this was attributed to a threshold effect. This is an expected finding in diagnostic test accuracy meta-analyses as the sensitivity and specificity both depend on the "threshold" or "cut-off" of determining the positivity of a test. Lowering the threshold on PSMA-PET for determining EPE would theoretically increase sensitivity at the cost of decreased specificity (or increased false-positive rate). Unlike in studies evaluating SVI, the anatomical imaging modality was not a factor of heterogeneity. However, when looking in detail at the subgroup of studies assessing EPE using PET/MRI and PET/CT, the summary sensitivity and specificity estimates were substantially different ( 0.82 vs. 0.65 and 0.73 vs. 0.95 for PET/MRI vs PET/CT, respectively) with wide and overlapping 
confidence intervals. These findings, along with the fact that there were only a small number of studies $(n=8)$ evaluating the performance of PSMA-PET for EPE, implicate that strong conclusions cannot be drawn.

The technical details for acquisition of PSMA-PET and its interpretation varied widely among the included studies. First, regarding the PSMA-targeting ligand, most studies used ${ }^{68}$ Ga-PSMA-11, one study used ${ }^{68}$ Ga-PSMA-I\&T. Furthermore, although we only assessed ${ }^{68} \mathrm{Ga}$-based radioligands, there are other newer non- ${ }^{68} \mathrm{Ga}$-based radioligands, which show promising results. For example, one comparative study for ${ }^{18}$ F-PSMA-1007 and ${ }^{68}$ Ga-PSMA-11 in 16 patients showed that ${ }^{18}$ F-PSMA-1007 may potentially have higher detectability for low-grade cancer (Gleason grade 3) than ${ }^{68} \mathrm{Ga}$ PSMA-11 (Kuten et al. 2020). In another study that included 7 patients, ${ }^{18}$ F-PSMA1007 PET/MRI yielded a sensitivity and specificity of $100 \%$ each for determining SVI. The main difference between ${ }^{18} \mathrm{~F}$-PSMA-1007 and ${ }^{68} \mathrm{Ga}$-PSMA- 11 is the reduced renal excretion of the ${ }^{18} \mathrm{~F}$-labeled compound, a potential benefit for local staging in close proximity to the bladder. Additional PSMA tracers (e.g., ${ }^{18}$ F-DCFBC, ${ }^{18}$ F-DCFPyL) are already currently being used, and as newer tracers are being developed, future studies are needed to investigate whether differences in PSMA tracers will result in different diagnostic capability (Walker et al. 2020). There was also wide variability in the injected radiopharmaceutical dose, uptake, and image acquisition time and usage of diuretics, which can also potentially affect the performance of PSMA-PET (Derlin et al. 2016). Even more importantly, most of the studies did not define set criteria for assessing SVI and EPE regarding each of the components of PSMA-PET and MRI/CT, along with how to perform an integrated interpretation of them. For MRI, several criteria for assessment of EPE (e.g., ESUR criteria, Mehralivand grading system, and length of tumor capsular contact) have been tested and validated in some studies (Mehralivand et al. 2019; Barentsz et al. 2012; Kim et al. 2020). Furthermore, recent efforts by multidisciplinary international group of experts, focused on proposing a standardized assessment of PSMA-PET with the molecular imaging TNM system (miTNM, version 1.0) and PSMA reporting and data systems (PSMA-RADS) version 1.0; however, these systems have neither been tested nor validated in the literature (Eiber et al. 2018; Rowe et al. 2018). Nevertheless, the promising results in the current meta-analysis despite the lack of standardization of image acquisition and interpretation show not only the high potential of PSMA-PET for local staging but also the need for clear and validated criteria for the performance and interpretation of PSMA-PET for local staging. These criteria will need to (1) define the roles of PET and CT/MRI for determining disease extent and to (2) address how these functional and anatomical imaging components can be interpreted together. This could also help improve the inter-reader agreement which was only fair in one of the included studies by Muehleamatter et al. (Muehlematter et al. 2019) that assessed it (kappa of 0.33 for SVI and 0.40 for EPE), and accelerate its widespread adoption.

There are some limitations in this meta-analysis. The number of included studies was small ( $n=12$ for SVI and 8 for EPE, respectively). Notwithstanding, this is currently the largest collective data providing a summary estimate of the performance of PSMAPET in determining the local extent of disease in prostate cancer in the setting of initial staging. In addition, even with the small number of studies, we were able to identify meaningful sources of heterogeneity that may have clinical implications. Second, all but 
two were retrospective single-center studies and therefore have the potential of inherent bias. Prospective multicenter studies may be needed to validate the reported diagnostic performance. Third, nearly all patients included had clinically intermediateto-high risk prostate cancer, and our results may not translate to those with low-risk disease. Fourth, as we used radical prostatectomy as the reference standard, the results are not directly applicable to patients receiving other treatments (e.g., active surveillance, focal treatment, radiation treatment, or systemic treatment). Fifth, EPE was not stratified by extent in all of the included studies. Investigators have observed trends of increasing sensitivity of MRI in detecting EPE with more extensive degrees of EPE. There may be value in designing future studies on PSMA-PET with assessment of EPE and SVI stratified to extent (e.g., $<1 \mathrm{~mm}, 1-2 \mathrm{~mm}$, and $>2 \mathrm{~mm}$ ) (Jager et al. 1996; Rosenkrantz et al. 2013).

\section{Conclusion}

PSMA-PET has moderate sensitivity and excellent specificity for assessing the local extent of the tumor in patients with intermediate to high-risk prostate cancer. PET/MRI showed potential for greater sensitivity than PET/CT in assessing SVI. Standardization of image acquisition and interpretation is needed to increase applicability and implementation of our results.

Code availability

No complex statistical codes or software were used. All simple codes were based on STATA and R, which for the latter all codes are freely available and accessible online. No custom codes were used.

\section{Authors' contributions}

All authors contributed to the study conception and design. Material preparation, data collection, and analysis were performed by Sungmin Woo, Soleen Ghafoor, and Hebert Alberto Vargas. The first draft of the manuscript was written by Sungmin Woo and all authors commented on previous versions of the manuscript. All authors read and approved the final manuscript.

Funding

This research was funded in part through the NIH/NCI Cancer Center Support Grant P30 CA008748. However, all data access and analysis were done solely by the authors of this study.

\section{Availability of data and materials}

All data was based on the 12 included papers that are accessible from either the journal webpages or by using Pubmed and EMBASE databases, some of which require journal subscription fees.

Ethics approval and consent to participate

Not applicable as this was a systematic review and meta-analysis.

\section{Consent for publication}

Not applicable as this was a systematic review and meta-analysis.

\section{Competing interests}

Hedvig Hricak has served on the Board of Directors of Ion Beam Applications (IBA), a publicly-traded company, and she receives annual compensation for her service since May 2017. Furthermore, Hedvig Hricak is a member of the External Advisory Board of the University of Michigan Comprehensive Cancer Center, the International Advisory Board of the University of Vienna, Austria, and the Scientific Committee of the DKFZ (German Cancer Research Center), Germany; she does not receive financial compensation for any of these roles. None of the above were related to the current study. The other authors do not have any other conflict of interest to disclose.

\section{Author details}

'Department of Radiology, Memorial Sloan Kettering Cancer Center, 1275 York Avenue, New York, NY 10065, USA.

${ }^{2}$ Department of Nuclear Medicine, Asan Medical Center, University of Ulsan College of Medicine, 88 Olympic-ro 43-gil, Songpa-gu, Seoul 05505, Korea. ${ }^{3}$ Department of Nuclear Medicine, University Hospital Zürich, University of Zürich,

Zürich, Switzerland. ${ }^{4}$ Department of Nuclear Medicine, Kantonsspital Baden, Baden, Switzerland. 
Received: 11 May 2020 Accepted: 5 August 2020

Published online: 09 September 2020

\section{References}

Agrawal A, Rangarajan V, Bakshi G, Prakash G, Menon S, Purandare N et al (2017) Can 68Ga-PSMA PET/CT predict seminal vesicle invasion for patients undergoing radical prostatectomy? Eur J Nucl Med Mol Imaging. 44:S369-SS70

Barentsz JO, Richenberg J, Clements R, Choyke P, Verma S, Villeirs G et al (2012) ESUR prostate MR guidelines 2012. Eur Radiol. 22:746-757

Berger I, Annabattula C, Lewis J, Shetty DV, Kam J, Maclean F et al (2018) (68)Ga-PSMA PET/CT vs. mpMRI for locoregional prostate cancer staging: correlation with final histopathology. Prostate Cancer Prostatic Dis. 21:204-211

Bostwick DG, Pacelli A, Blute M, Roche P, Murphy GP (1998) Prostate specific membrane antigen expression in prostatic intraepithelial neoplasia and adenocarcinoma: a study of 184 cases. Cancer. 82:2256-2261

Bray F, Ferlay J, Soerjomataram I, Siegel RL, Torre LA, Jemal A (2018) Global cancer statistics 2018: GLOBOCAN estimates of incidence and mortality worldwide for 36 cancers in 185 countries. CA Cancer J Clin. 68:394-424

Corfield J, Perera M, Bolton D, Lawrentschuk N (2018) (68)Ga-prostate specific membrane antigen (PSMA) positron emission tomography (PET) for primary staging of high-risk prostate cancer: a systematic review. World J Urol. 36:519-527

de Rooij M, Hamoen EHJ, Witjes JA, Barentsz JO, Rovers MM (2016) Accuracy of magnetic resonance imaging for local staging of prostate cancer: a diagnostic meta-analysis. Eur Urol. 70:233-245

Deeks JJ, Macaskill P, Irwig L (2005) The performance of tests of publication bias and other sample size effects in systematic reviews of diagnostic test accuracy was assessed. J Clin Epidemiol. 58:882-893

Dekalo S, Kuten J, Mabjeesh NJ, Beri A, Even-Sapir E, Yossepowitch O (2019) 68Ga-PSMA PET/CT: does it predict adverse pathology findings at radical prostatectomy? Urol Oncol 37:574.e19-574.e24

Derlin T, Weiberg D, von Klot C, Wester H-J, Henkenberens C, Ross TL et al (2016) (68)Ga-PSMA I\&T PET/CT for assessment of prostate cancer: evaluation of image quality after forced diuresis and delayed imaging. Eur Radiol 26:4345-4353

Eiber M, Herrmann K, Calais J, Hadaschik B, Giesel FL, Hartenbach M et al (2018) Prostate cancer molecular imaging standardized evaluation (PROMISE): proposed miTNM classification for the interpretation of PSMA-ligand PET/CT. J NUCl Med. 59:469-478

Eiber M, Weirich G, Holzapfel K, Souvatzoglou M, Haller B, Rauscher I et al (2016) Simultaneous (68)Ga-PSMA HBED-CC PET/ MRI improves the localization of primary prostate cancer. Eur Urol. 70:829-836

Fendler WP, Calais J, Eiber M, Flavell RR, Mishoe A, Feng FY et al (2019) Assessment of 68Ga-PSMA-11 PET accuracy in localizing recurrent prostate cancer: a prospective single-arm clinical trial. JAMA Oncol. 5:856-863

Fendler WP, Schmidt DF, Wenter V, Thierfelder KM, Zach C, Stief C et al (2016) 68Ga-PSMA PET/CT detects the location and extent of primary prostate cancer. J Nucl Med. 57:1720-1725

Gao J, Zhang C, Zhang Q, Fu Y, Zhao X, Chen M et al (2019) Diagnostic performance of (68)Ga-PSMA PET/CT for identification of aggressive cribriform morphology in prostate cancer with whole-mount sections. Eur J Nucl Med Mol Imaging. 46:1531-1541

Grubmuller B, Baltzer P, Hartenbach S, D'Andrea D, Helbich TH, Haug AR et al (2018) PSMA ligand PET/MRI for primary prostate cancer: staging performance and clinical impact. Clin Cancer Res. 24:6300-6307

Gupta M, Choudhury PS, Rawal S, Goel HC, Talwar V, Singh A et al (2018) Initial risk stratification and staging in prostate cancer with prostatic-specific membrane antigen positron emission tomography/computed tomography: a first-stopshop. World J Nucl. 17:261-269

Han S, Woo S, Kim YJ, Suh CH (2018) Impact of (68)Ga-PSMA PET on the management of patients with prostate cancer: a systematic review and meta-analysis. Eur Urol. 74:179-190

Hicks RM, Simko JP, Westphalen AC, Nguyen HG, Greene KL, Zhang L et al (2018) Diagnostic accuracy of (68)Ga-PSMA-11 PET/MRI compared with multiparametric MRI in the detection of prostate cancer. Radiology. 289:730-737

Higgins J, Green S. Cochrane handbook for systematic reviews of interventions version 5.1.0. The Cochrane Collaboration. 2011 http://handbook.cochrane.org/chapter_9/9_5_2_identifying_and_measuring_heterogeneity.htm. . Accessed 3 Jan 2017.

Hofman MS, Hicks RJ, Maurer T, Eiber M (2018) Prostate-specific membrane antigen PET: clinical utility in prostate cancer, normal patterns, pearls, and pitfalls. Radiographics. 38:200-217

Jager GJ, Ruijter ET, van de Kaa CA et al (1996) Local staging of prostate cancer with endorectal MR imaging: correlation with histopathology. AJR Am J Roentgenol. 166(4):845-852

Jansen BHE, Nieuwenhuijzen JA, Oprea-Lager DE, Yska MJ, Lont AP, van Moorselaar RJA et al (2019) Adding multiparametric MRI to the MSKCC and Partin nomograms for primary prostate cancer: improving local tumor staging? Urol Oncol 37: 181.e1-181.e6

Kim TH, Woo S, Han S, Suh CH, Ghafoor S, Hricak H et al (2020) The diagnostic performance of the length of tumor capsular contact on magnetic resonance imaging for detecting prostate cancer extraprostatic extension: a systematic review and meta-analysis. Korean J Radiol. 21:e69

Kuten J, Fahoum I, Savin Z, Shamni O, Gitstein G, Hershkovitz D et al (2020) Head- to head comparison of (68)Ga-PSMA-11 with (18)F-PSMA-1007 PET/CT in staging prostate cancer using histopathology and immunohistochemical analysis as reference-standard. J Nucl Med. 61:527-532

Lee J, Kim KW, Choi SH, Huh J, Park SH (2015) Systematic review and meta-analysis of studies evaluating diagnostic test accuracy: a practical review for clinical researchers-Part II. Statistical methods of meta-analysis. Korean J Radiol. 16:11881196

Liberati A, Altman DG, Tetzlaff J, Mulrow C, Gøtzsche PC, loannidis JPA et al (2009) The PRISMA statement for reporting systematic reviews and meta-analyses of studies that evaluate health care interventions: explanation and elaboration. J Clin Epidemiol. 62:e1-e34

Mehralivand S, Shih JH, Harmon S, Smith C, Bloom J, Czarniecki M et al (2019) A grading system for the assessment of risk of extraprostatic extension of prostate cancer at multiparametric MRI. Radiology. 290:709-719. https://doi.org/10.1148/radiol. 2018181278 
Mikel Hubanks J, Boorjian SA, Frank I, Gettman MT, Houston Thompson R, Rangel LJ et al (2014) The presence of extracapsular extension is associated with an increased risk of death from prostate cancer after radical prostatectomy for patients with seminal vesicle invasion and negative lymph nodes. Urol Oncol 32:26. e1-26. e7

Mottet N, Bellmunt J, Bolla M, Briers E, Cumberbatch MG, De Santis M et al (2017) EAU-ESTRO-SIOG guidelines on prostate cancer. Part 1: screening, diagnosis, and local treatment with curative intent. Eur Urol. 71:618-629

Mottet N, van den Bergh RCN, Briers E, Cornford P, De Santis M, Fanti S, et al. EAU - ESTRO - ESUR - SIOG guidelines on prostate cancer 2020. Retrieved from: https://uroweb.org/guideline/prostate-cancer/ Access date 7 May 2020.

Muehlematter UJ, Burger IA, Becker AS, Schawkat K, Hotker AM, Reiner CS et al (2019) Diagnostic accuracy of multiparametric MRI versus (68)Ga-PSMA-11 PET/MRI for extracapsular extension and seminal vesicle invasion in patients with prostate cancer. Radiology. 293:350-358

Nyarangi-Dix J, Wiesenfarth M, Bonekamp D, Hitthaler B, Schütz V, Dieffenbacher S et al (2018) Combined clinical parameters and multiparametric magnetic resonance imaging for the prediction of extraprostatic disease-a risk model for patienttailored risk stratification when planning radical prostatectomy. Eur Urol Focus S2405-4569(18):30336-30335

Ohori M, Kattan MW, Koh H, Maru N, Slawin KM, Shariat S et al (2004) Predicting the presence and side of extracapsular extension: a nomogram for staging prostate cancer. J Urol. 171:1844-1849

Park K, Kim MH, Kim JK (2020) Extraprostatic tumor extension: comparison of preoperative multiparametric MRI criteria and histopathologic correlation after radical prostatectomy. Radiology. 192133. https://doi.org/10.1148/radiol.2020192133

Park SY, Zacharias C, Harrison C, Fan RE, Kunder C, Hatami N et al (2018) Gallium 68 PSMA-11 PET/MR imaging in patients with intermediate- or high-risk prostate cancer. Radiology. 288:495-505

Perera M, Papa N, Roberts M, Williams M, Udovicich C, Vela I et al (2020) Gallium-68 prostate-specific membrane antigen positron emission tomography in advanced prostate cancer-updated diagnostic utility, sensitivity, specificity, and distribution of prostate-specific membrane antigen-avid lesions: a systematic review and meta-analysis. Eur Urol. 77:403417

Rosenkrantz AB, Chandarana H, Gilet A et al (2013) Prostate cancer: utility of diffusion-weighted imaging as a marker of sidespecific risk of extracapsular extension. J Magn Reson Imaging. 38(2):312-319

Rowe SP, Pienta K, Pomper MG, Gorin MA (2018) PSMA-RADS Version 1.0: a step towards standardizing the interpretation and reporting of PSMA-targeted PET imaging studies. Eur Urol 73:485-487

Suh CH, Park SH (2016) Successful publication of systematic review and meta-analysis of studies evaluating diagnostic test accuracy. Korean J Radiol. 17:5-6

Thalgott M, Duwel C, Rauscher I, Heck MM, Haller B, Gafita A et al (2018) One-stop-shop whole-body (68)Ga-PSMA-11 PET/ MRI compared with clinical nomograms for preoperative T and N staging of high-risk prostate cancer. J Nucl Med. 59: 1850-1856

van Leeuwen PJ, Donswijk M, Nandurkar R, Stricker P, Ho B, Heijmink S et al (2019) Gallium-68-prostate-specific membrane antigen ((68) Ga-PSMA) positron emission tomography (PET)/computed tomography (CT) predicts complete biochemical response from radical prostatectomy and lymph node dissection in intermediate- and high-risk prostate cancer. BJU Int. 124:62-68

von Klot CJ, Merseburger AS, Boker A, Schmuck S, Ross TL, Bengel FM et al (2017) (68)Ga-PSMA PET/CT imaging predicting intraprostatic tumor extent, extracapsular extension and seminal vesicle invasion prior to radical prostatectomy in patients with prostate cancer. Nucl Med Mol Imaging. 51:314-322

Walker SM, Lim I, Lindenberg L, Mena E, Choyke PL, Turkbey B (2020 [Online ahead of print]) Positron emission tomography (PET) radiotracers for prostate cancer imaging. Abdom Radiol (NY). https://doi.org/10.1007/s00261-020-2427-4

Wang L, Hricak H, Kattan MW, Chen HN, Kuroiwa K, Eisenberg HF et al (2007) Prediction of seminal vesicle invasion in prostate cancer: incremental value of adding endorectal MR imaging to the Kattan nomogram. Radiology. 242:182-188

Weaver JK, Kim EH, Vetter JM, Shetty A, Grubb RL 3rd, Strope SA et al (2018) Prostate magnetic resonance imaging provides limited incremental value over the Memorial Sloan Kettering Cancer Center preradical prostatectomy nomogram. Urology. 113:119-128

Whiting PF, Rutjes AW, Westwood ME, Mallett S, Deeks JJ, Reitsma JB et al (2011) QUADAS-2: a revised tool for the quality assessment of diagnostic accuracy studies. An Intern Med. 155:529-536

Yilmaz B, Turkay R, Colakoglu Y, Baytekin HF, Ergul N, Sahin S et al (2019) Comparison of preoperative locoregional Ga-68 PSMA-11 PET-CT and mp-MRI results with postoperative histopathology of prostate cancer. Prostate. 79:1007-1017

Zhou J, Gou Z, Wu R, Yuan Y, Yu G, Zhao Y (2019) Comparison of PSMA-PET/CT, choline-PET/CT, NaF-PET/CT, MRI, and bone scintigraphy in the diagnosis of bone metastases in patients with prostate cancer: a systematic review and meta-analysis. Skeletal Radiol. 48:1915-1924

\section{Publisher's Note}

Springer Nature remains neutral with regard to jurisdictional claims in published maps and institutional affiliations. 